\title{
Patrones Morfológicos Asociados a Factores de Riesgo Metabólico en Población de Adolescentes Escolarizados
}

\author{
Morphological Patterns Associated with Metabolic \\ Risk Factors in the Adolescent School Population
}

Espinoza-Navarro,O. ${ }^{1}$ \& Brito-Hernández, L. $^{2}$

ESPINOZA-NAVARRO,O. \& BRITO-HERNÁNDEZ, L. Patrones morfológicos asociados a factores de riesgo metabólico en población de adolescentes escolarizados. Int. J. Morphol., 38(6):1645-1650, 2020.

RESUMEN: El aumento sostenido en la prevalencia de sobrepeso y obesidad en niños, niñas y adolescentes, causa alarma en la comunidad científica, que observa incrementos importantes en las enfermerdades asociadas a Síndrome Metabólico (SM), en la vida adulta. Chile presenta un $75 \%$ con sobrepeso y obesidad en la población adulta y un 50,9 \% en la población estudiantil, con un 66,2 \% de sedentarismo. Los objetivos de este trabajo fueron determinar los perfiles antropométricos y su asociación a riesgo metabólico en adolescentes de colegio particular subvencionado de Arica-Chile. El total de los alumnos de enseñanza media, $n=810$ (mujeres $n=437$ y hombres $n=373$ ), fueron evaluados según: peso, talla, perímetro de cintura, índice cintura-talla (ICT), índice de masa corporal (IMC). A todos ellos se les calculó el riesgo metabólico según protocolos de Ashwell \& Gibson (2016) y el IMC según fórmula de Quetelet. Los datos fueron analizados en estadístico para tendencia central, dispersión y porcentajes. Para correlación se utilizó test de Pearson (r>0,5). Los resultados muestran que un $44,1 \%$ de las mujeres y un $37,2 \%$ de los hombres presentan sobrepeso y obesidad. El riesgo metabólico general de la población en estudio fue de $24,6 \%$ (24,7 \% y 24,4 \% en mujeres y hombres respectivamente). Se observó una alta correlación entre PC / ICT ( $\mathrm{r}=0,92)$, IMC / ICT $(\mathrm{r}=0,86)$ y Peso / PC $(\mathrm{r}=0,87)$. Se concluye que las y los adolescentes presentan valores antropométricos alterados que indican altos índices de riesgos metabólicos. Los parámetros más alterados se observan en el segundo año de enseñanza media con porcentajes de sobrepeso y obesidad de 49,1\% en las mujeres versus 33,8 \% en los hombres. Independientemente, los hombres presentaron mejores índices morfométricos que las mujeres. Se observaron altas correlaciones $(r>0,5)$ en todos los parámetros en estudio, asociados a riesgo metabólico. Se sugiere enfocar las intervenciones educativas según sello de vida saludable, incentivando la actividad física y mejorar los hábitos alimenticios en las y los adolescentes escolarizados.

PALABRAS CLAVE: Educación; Riesgo Metabólico; Adolescentes; Sedentarismo Chile.

\section{INTRODUCCIÓN}

En las últimas décadas las patologías cardiovasculares asociadas a sobrepeso y obesidad, han aumentado las tasas de mortalidad en un $60 \%$ (OMS, 2016; Espinoza-Navarro et al., 2020). Los estudios epidemiológicos han demostrado que se trata de un proceso gradual que se inicia a partir de la primera o segunda década de vida, expresando manifestaciones clínicas de riesgo en las edades posteriores (MeraGallego et al., 2016).

Las prevalencias de sobrepeso y obesidad y sus riesgos asociados en población estudiantil chilena, muestran un alza creciente, los registros actuales en estudiantes de enseñanza básica presentan valores de sobrepeso y obesidad de un $52,5 \%$ y de riesgo metabólico de un $35 \%$ (BritoHernández et al., 2018).
En Chile las políticas educativas de Enseñanza Básica, orientan sus objetivos en la adquisición de conocimientos, hábitos de postura, higiene y salud en sus educandos. En cambio en la Enseñanza Media los objetivos se centran en el conocimiento (desarrollo de la inteligencia) y en los valores (MINEDUC, 2018), este tipo de educación concentra a la población adolescente, desde los 14 años a los 18 años.

Según la Organización Mundial de la Salud (OMS, 2014), la población adolescente comprende un periodo de entre los 10 años a los 19 años, corresponde a una etapa muy compleja que marca la transición de la infancia al estado adulto, enfrentando cambios biomorfológicos, psiciológicos, intelectuales y sociales. Estos cambios facilitan la instalación de factores de riesgo cardimetabólico con un efecto multiplicativo

\footnotetext{
${ }^{1}$ Laboratorio de Reproducción y Desarrollo, Departamento de Biología, Universidad de Tarapacá, Arica, Chile.

${ }^{2}$ North American College, Laboratorio Biología del Desarrollo, Universidad de Tarapacá, Arica, Chile.
} 
más que sumativo, sobre todo en la variación de la cantidad y de la localización de la grasa corporal, acelerando las patologías propias del síndrome metabólico (SM), como diabetes y ateroesclerosis (Cordero et al., 2015; Reynoso Vázquez et al., 2018). Otros autores relacionan la expresión del síndrome metabólico, en adolescentes con patrones antropómétricos, sedentarismo, alimentación y estilo de vida.

Burrows et al. (2016) relacionan el índice de masa corporal (IMC), circunferencia de cintura (CC), masa grasa y parámetros bioquímicos, con una alta prevalencia de obesidad, dislipidemia, hiperglicemia y síndrome metabólico en los adolescentes. Estudios en adolescentes escolarizados de México y Venezuela, muestran las mismas correlaciones (VillegasSepúlveda et al., 2016; Acosta-García \& Concepción-Páez, 2018). La misma literatura reporta que estas patologías son prevenibles con una dieta saludable y actividad física regular (Macedo-Uchôa et al., 2019). Leiva et al. (2018) recomiendan políticas en la Educación y en la Salud Pública Chilenas, que prioricen estilos de vida saludable en los estudiantes desde temprana edad. En estas políticas es imprescindible incorporar a todos los actores, la familia, los establecimientos y los profesores (Collipal \& Godoy, 2015; Espinoza-Navarro et al.). Específicamente el objetivo de este estudio fue determinar el perfil antropométrico y los factores de riesgo metabólico asociados, en población adolescente escolarizada de Chile.

\section{MATERIAL Y MÉTODO}

Este estudio corresponde a un diseño de tipo, descriptivo, transversal y correlacional. La población en estudio corresponde a 810 estudiantes de enseñanza media de un colegio particular subvencionado de Arica-Chile (437 mujeres, 373 hombres), en edades de entre 14 a 18 años, categorizados por niveles $\left(1^{\circ}, 2^{\circ}, 3^{\circ}\right.$ y $4^{\circ}$ medio), quienes aceptaron voluntariamente a participar. Luego de firmados los consentimientos informados por los apoderados y los asentimientos informados por los estudiantes, estos adolescentes fueron evaluados en el mismo establecimiento educacional, en horario de la mañana, con ropa ligera y sin zapatos. Se obtuvo el peso y talla, para calcular el índice de masa corporal (IMC), utilizando la fórmula de Quetelet (peso en kilógramos/talla en metros al cuadrado) (MINSAL, 2017). Luego se midió el perímetro de cintura (PC) y se calculó el índice cintura-talla (ICT). El riesgo metabólico se determinó utilizando el índice de cintura/talla según protocolos de Ashwell \& Gibson, con punto de corte 0,50 . Los instrumentos de medición utilizados fueron: Balanza con tallímetro portatil (ADE M3 18300, West Germany), con precisión de $100 \mathrm{~g}$ (peso) y $0,1 \mathrm{~cm}$ (talla). Los protocolos éticos del proyecto fueron aprobados por el Comité Ético Científico acreditado de la Universidad de Tarapacá.
Los datos fueron analizados en lenguaje de código abierto R. Luego se procedió a realizar descriptiva mediante estadísticos de tendencia central, dispersión, y porcentajes. Cuando fue necesario se realizó inferencia utilizando significancia $\mathrm{p}<0,05$ (t-test). Se realizaron gráficos de correlación entre métricas según estadístico de correlación de Pearson $(r>0,5)$.

\section{RESULTADOS}

Las Tablas de la I a la IV y la Figura 1, muestran los resultados antropométricos y los estudios de correlación obtenidos y su asociación con riesgo metabólico en esta población adolescente escolarizada $(\mathrm{n}=810)$, según sexo y niveles de enseñanza media.

En la Tabla I se observan los datos generales de los registros antropométricos, porcentajes de sobrepeso y obesidad y los porcentajes de riesgos metabólicos asociados. Los hombres presentan valores más altos en peso, talla y perímetro de cintura, respecto a las mujeres. ICT e IMC no muestran diferencias entre sexo. Las mujeres presentan valores muchos más elevados en sobrepeso $(30,7 \%)$, respecto a los hombres $(21 \%)$. El parámetro de obesidad presenta un valor general de $14,7 \%$, sin diferencias entre sexo. El riesgo metabólico asociado fue de un $24,6 \%$, sin diferencias significativas entre sexos.

La Tabla II muestra los registros antropométricos de la población según nivel educativo y sexo. Se observa una progresión natural en el transcurso de las edades, con cambios diferenciales mayores en los hombres. Diferencial peso (k): hombres $+9,24$ y mujeres $+1,62$. Diferencial perímetro cintura $(\mathrm{cm}):+3.76 /+0,84$ respectivamente.

La sumatoria de los registros de sobrepeso y obesidad se muestran en la Tabla III, donde las mujeres presentan valores generales de $44,1 \%$ y los hombres valores más bajos de $37,2 \%$ (diferencial de 6,9\%). El análisis por niveles, muestra que las mayores diferencias se presentan en el nivel de segundo medio, donde las mujeres presentan valores de $49,1 \%$ y los hombres $33,8 \%$, lo que expresa un diferencial de un 15,3 $\%$ de sobrepeso y obesidad. Todos los niveles presentan esta tendencia, pero con menores diferenciales.

En la Tabla IV, se muestran los porcentajes de riesgo metabólico en esta población de adolescentes, con valores generales para mujeres de $24,7 \%$ y para hombres de $24,4 \%$ respectivamente. Según el nivel de educación, se observa un alza significativa en mujeres de segundo medio con un $31,7 \%$ respecto a los hombres con $26,3 \%$, lo que expresa un diferencial de $5,4 \%$ de riesgo metabólico, en este nivel. 
ESPINOZA-NAVARRO,O. \& BRITO-HERNÁNDEZ, L. Patrones morfológicos asociados a factores de riesgo metabólico en población de adolescentes escolarizados. Int. J. Morphol., 38(6):1645-1650, 2020

Tabla I. Datos generales antropométricos y de riesgo metabólico de la población estudiantil de enseñanza media, $n=810$. Mujeres $n=437$; Hombres $n=373$. $P C=$ perímetro de cintura; $\mathrm{ICT}=$ índice cintura talla

\begin{tabular}{lccc}
\hline & GENERAL & MUJERES & HOMBRES \\
\cline { 2 - 4 } & $(\mathrm{n}=810)$ & $(\mathrm{n}=437)$ & $(\mathrm{n}=373)$ \\
\hline EDAD (años) & MEDIA \pm DS & MEDIA \pm DS & MEDIA \pm DS \\
PESO (k) & $15,4 \pm 1,26$ & $15,4 \pm 1,3$ & $15,4 \pm 1,3$ \\
TALLA (m) & $63,7 \pm 14,9$ & $61 \pm 13,4$ & $66,87 \pm 15,8$ \\
PC (cm) & $1,63 \pm 0,1$ & $1,59 \pm 0,06$ & $1,7 \pm 0,08$ \\
IMC & $75,2 \pm 10,9$ & $73,4 \pm 10,2$ & $77,23 \pm 11,2$ \\
ICT & 23,6 & 24,1 & 23,19 \\
SOBREPESO \% & $0,45 \pm 0,06$ & $0,46 \pm 0,06$ & $0,46 \pm 0,06$ \\
OBESIDAD \% & 27,5 & 30,7 & 21,0 \\
CON RIESGO METABÓLICO & 14,7 & 13,3 & 16,3 \\
\hline
\end{tabular}

Tabla II. Antropometría de la población estudiantil por nivel y sexo, n=810. Mujeres n= 437; Hombres $n=373$. PC $=$ perímetro de cintura; ICT= índice cintura talla.

\begin{tabular}{|c|c|c|c|c|c|c|}
\hline \multicolumn{7}{|c|}{ ANTROPOMETRÍA MUJERES } \\
\hline & EDAD (años) & PESO (k) & TALLA (m) & $\mathrm{PC}(\mathrm{cm})$ & IMC & ICT \\
\hline NIVEL (n) & Media \pm DS & Media \pm DS & Media \pm DS & Media \pm DS & Media \pm DS & Media \pm DS \\
\hline $1^{\circ} \operatorname{MEDIO}(n=111)$ & $13,92 \pm 0,52$ & $59,15 \pm 12,83$ & $1,59 \pm 0,06$ & $72,11 \pm 10,37$ & 23,4 & $0,45 \pm 0,06$ \\
\hline $2^{\circ} \operatorname{MEDIO}(n=120)$ & $14,97 \pm 0,56$ & $61,09 \pm 12,36$ & $1,58 \pm 0,05$ & $75,31 \pm 10,04$ & 24,2 & $0,47 \pm 0,06$ \\
\hline $3^{\circ} \operatorname{MEDIO}(n=105)$ & $16,04 \pm 0,78$ & $62,93 \pm 15,02$ & $1,60 \pm 0,06$ & $73,0 \pm 10,45$ & 24,6 & $0,46 \pm 0,06$ \\
\hline $4^{\circ} \operatorname{MEDIO}(n=101)$ & $16,84 \pm 0,61$ & $60,77 \pm 13,53$ & $1,59 \pm 0,06$ & $72,95 \pm 9,65$ & 23,9 & $0,46 \pm 0,06$ \\
\hline DIFERENCIAL & & $+1,62$ & & $+0,84$ & $+0,5$ & $+0,01$ \\
\hline \multicolumn{7}{|c|}{ ANTROPOMETRÍA HOMBRES } \\
\hline NIVEL (n) & Media \pm DS & Media \pm DS & Media \pm DS & Media \pm DS & Media \pm DS & Media \pm DS \\
\hline $1^{\circ} \operatorname{MEDIO}(\mathrm{n}=111)$ & $13,96 \pm 0,56$ & $62,62 \pm 15,42$ & $1,66 \pm 0,07$ & $75,28 \pm 11,77$ & 22,6 & $0,45 \pm 0,07$ \\
\hline $2^{\circ} \operatorname{MEDIO}(n=120)$ & $14,98 \pm 0,53$ & $65,52 \pm 15,51$ & $1,70 \pm 0,06$ & $77,33 \pm 10,79$ & 22,9 & $0,46 \pm 0,06$ \\
\hline $3^{\circ} \operatorname{MEDIO}(n=105)$ & $16,03 \pm 0,69$ & $68,55 \pm 15,41$ & $1,72 \pm 0,07$ & $77,82 \pm 10,91$ & 23,2 & $0,45 \pm 0,06$ \\
\hline $4^{\circ} \operatorname{MEDIO}(n=101)$ & $16,96 \pm 9,52$ & $71,86 \pm 16,04$ & $1,72 \pm 0,06$ & $79,04 \pm 11,28$ & 24,1 & $0,46 \pm 0,06$ \\
\hline DIFERENCIAL & & $+9,24$ & $+0,06$ & $+3,76$ & $+1,5$ & $+0,01$ \\
\hline
\end{tabular}

Tabla III. Sumatoria de los porcentajes de sobrepeso y obesidad en la población estudiantil, según sexo y nivel.

\begin{tabular}{ccccccccc}
\hline & \multicolumn{7}{c}{ SOBREPESO Y OBESIDAD } \\
& MUJERES & \multicolumn{7}{c}{ HOMBRES } \\
\hline & $\mathrm{N}$ & $\mathrm{n}$ & $\mathbf{\%}$ & $\mathrm{N}$ & $\mathrm{n}$ & $\mathbf{\%}$ & Total & Diferencial \% \\
\hline $1^{\mathbf{0}}$ MEDIO & 111 & 49 & $\mathbf{4 4 , 1}$ & 109 & 46 & $\mathbf{4 2 , 2}$ & 220 & 1,9 \\
$2^{\mathbf{0}}$ MEDIO & 120 & 59 & $\mathbf{4 9 , 1}$ & 80 & 27 & $\mathbf{3 3 , 8}$ & 200 & 15,3 \\
$3^{\mathbf{0}}$ MEDIO & 105 & 49 & $\mathbf{4 6 , 6}$ & 105 & 42 & $\mathbf{4 0 , 0}$ & 210 & 6,6 \\
$4^{\mathbf{0}}$ MEDIO & 101 & 36 & $\mathbf{3 5 , 6}$ & 79 & 24 & $\mathbf{3 0 , 4}$ & 180 & 5,2 \\
TOTAL & 437 & 193 & $\mathbf{4 4 , 1}$ & 373 & 139 & $\mathbf{3 7 , 2}$ & 810 & 6,9 \\
\hline
\end{tabular}

Tabla IV. Porcentajes de riesgo metabólico en la población estudiantil de enseñanza media en mujeres y hombres, según nivel educativo.

\begin{tabular}{ccccccccc}
\hline \multicolumn{7}{c}{$\begin{array}{c}\text { ADOLESCENTES CON RIESGO METABÓLICO } \\
\text { HUJERES }\end{array}$} & \multicolumn{7}{c}{ HOMBRES } & \\
\hline & $\mathrm{N}$ & $\mathrm{n}$ & $\%$ & $\mathrm{~N}$ & $\mathrm{n}$ & $\%$ & Total & Diferencial \% \\
\hline $1^{\mathbf{o}}$ MEDIO & 111 & 26 & $\mathbf{2 3 , 4}$ & 109 & 29 & $\mathbf{2 6 , 6}$ & 220 & $-3,2$ \\
$2^{\mathbf{0}}$ MEDIO & 120 & 38 & $\mathbf{3 1 , 7}$ & 80 & 21 & $\mathbf{2 6 . 3}$ & 200 & $+5,4$ \\
$3^{\mathbf{0}}$ MEDIO & 105 & 20 & $\mathbf{1 9}$ & 105 & 22 & $\mathbf{2 1}$ & 210 & -3 \\
$4^{\mathbf{o}}$ MEDIO & 101 & 24 & $\mathbf{2 3 , 8}$ & 79 & 19 & $\mathbf{2 4}$ & 180 & $-0,2$ \\
TOTAL & 437 & 108 & $\mathbf{2 4 , 7}$ & 373 & 91 & $\mathbf{2 4 , 4}$ & 810 & $-0,03$ \\
\hline
\end{tabular}


ESPINOZA-NAVARRO,O. \& BRITO-HERNÁNDEZ, L. Patrones morfológicos asociados a factores de riesgo metabólico en población de adolescentes escolarizados. Int. J. Morphol., 38(6):1645-1650, 2020

En el Figura 1, se muestran las correlaciones entre las variables de peso (P), IMC, PC, ICT. Todas los gráficos
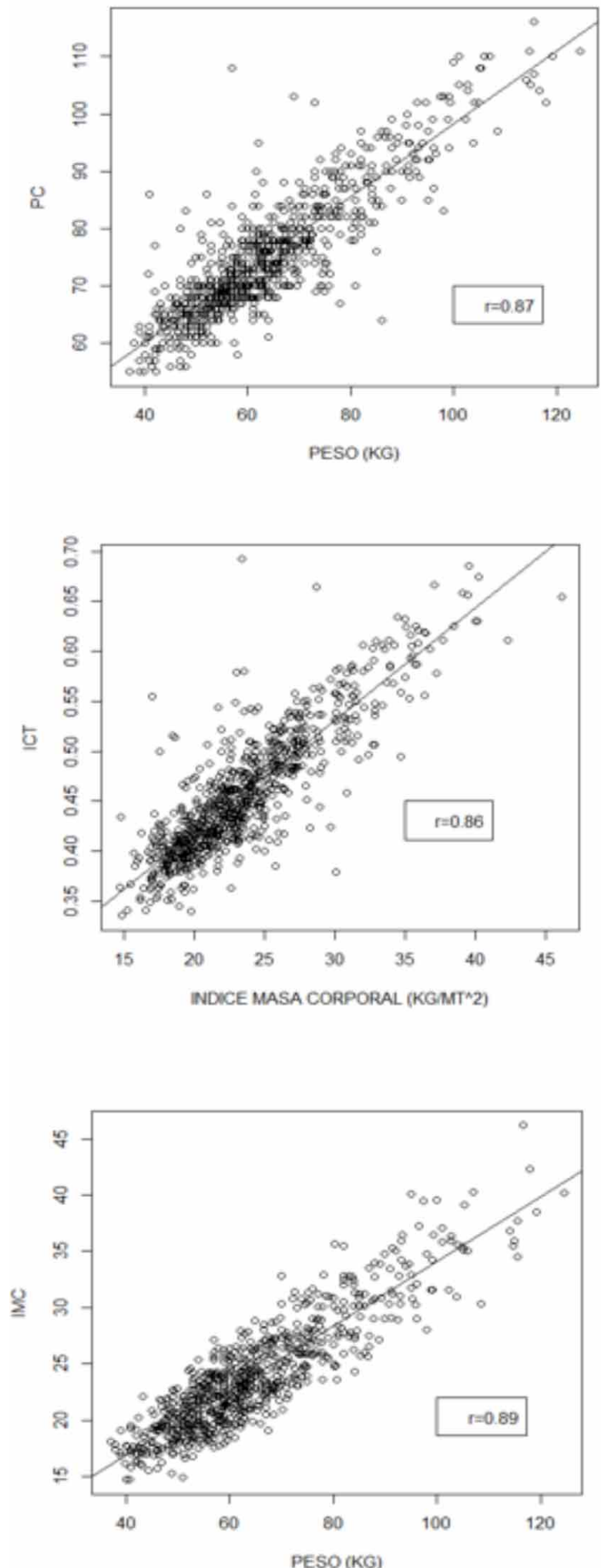

muestran una alta correlación directa entre las variables, con valores de $r>0,72$ a $r>0,92$ (valor absoluto $r>0,5$ ).
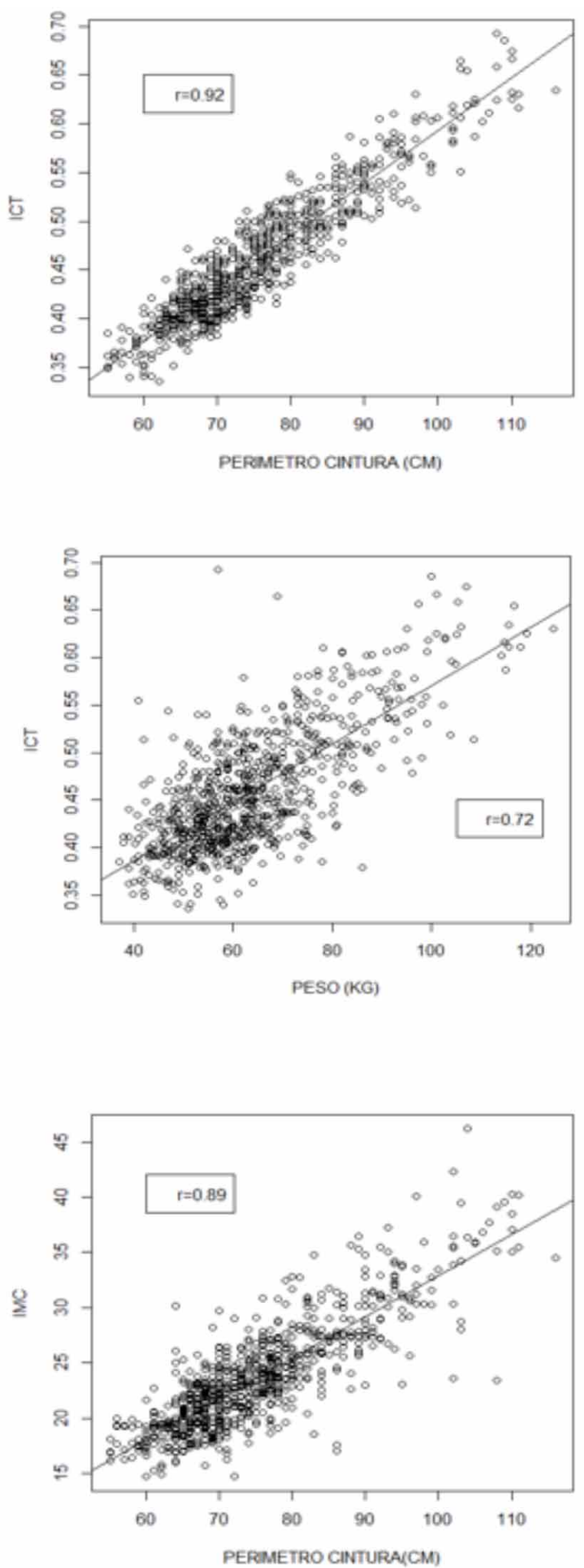

Fig. 1. Correlaciones entre las diferentes variables. Coeficiente de correlación (valor absoluto $r>0.5$ ). 


\section{DISCUSIÓN}

Factores demográficos, socioeconómicos y estilos de vida, han determinado importantes cambios en las conductas alimentarias y de actividad física en la población chilena, incrementando los niveles de sobrepeso y obesidad (Atalah, 2012). La Encuesta Nacional de Salud (MINSAL), registra para la población general chilena, niveles de sobrepeso de un $71 \%$ y de obesidad mórbida de un 3,2\% que se asocian a un riesgo metabólico de un 40,1 \% (13,6\% para jóvenes de entre 15 a 24 años). El sobrepeso y obesidad en la población adolescente supone la aparición de comorbilidades cardiovasculares, endocrino-metabólico, tumorales y psicosociales con un aumento, de riesgo de mortalidad precoz (Martínez-Villanueva, 2017).

Los resultados recopilados en esta investigación muestran niveles de sobrepeso de un 27,5\% y de obesidad de un $14,7 \%$, con riesgo metabólico asociado de un $24,6 \%$ (Tablas I, III y IV). Los registros de estos valores son más elevados que los promedios chilenos y de otras poblaciones mundiales de adolescentes. Villegas-Sepúlveda et al., reportan valores menores de sobrepeso y obesidad con un $24,7 \%$ y un $7 \%$ respectivamente, en jóvenes mexicanos. Similares resultados presentan Cordero et al. en estudiantes venezolanos, quienes además informan un riesgo metabólico asociado de solo un $10 \%$. Ramos-Padilla et al. (2015), informan registros similares en escolares ecuatorianos. Mera-Gallego et al., en población adolescente española, reportan valores de sobrepeso en mujeres de $23,6 \%$ y en hombres de $12,7 \%$, con registros de obesidad de $12,7 \%$ y $5 \%$, respectivamente.

La Tabla II muestra diferenciales naturales en la progresión etarea de adolescentes mujeres y hombres, donde los hombres presentan mayores diferenciales en peso corporal (+ $9,24 \mathrm{k})$, Talla $(+0,06 \mathrm{~m})$ y perímetro de cintura $(3,76 \mathrm{~cm})$, este comportamiento se repite en la Tabla III, donde las mujeres registran mayores porcentajes de sobrepeso y obesidad, sobre todo en segundo nivel de enseñanza media con diferenciales de $+15,3 \%$. Iglesias Diz (2013), atribuye este comportamiento en los adolescentes, a cambios hormonales, con producción de testosterona en los hombres, que favorece el crecimiento lineal y la lipolisis, indicando además que la presencia de síndrome metabólico en la pubertad puede condicionar la fertilidad durante la edad adulta.

Los Modelos antropométricos resultan de gran valor para contrastar y correlacionar los cambios o modificaciones en la morfoestructura y la fisiología humana como predictores del riesgo cardiometabólico. Los resultados observados en el Figura 1, muestran altas correlaciones positivas en todas las variables en estudio, siendo las más altas, las correlaciones de PC/ICT $(r=0,92)$ y de IMC con peso y $\mathrm{PC}(\mathrm{r}=0,89)$. Estas correlaciones reafirman la directa relación de la mediciones antropométricas y la asociación con riesgo cardiometabólico (Ashwell \& Gibson; Huamán et al., 2017; Díaz et al., 2017). La incorporación de las mediciones morfológicas permiten la identificación precoz de transtornos y patologías componentes del síndrome metabólico, permitiendo mejores intervenciones en los adolescente (Guszek et al., 2020).

Leiva et al. declaran la importancia de controlar el gasto energético y el peso corporal, implementando estilos de vida saludables, con incorporación de actividad física, que podrían bajar los factores de riesgo cardiometabólico, aumentando la esperanza de vida en 07 años o más años, esta incentivación hacia estos estilos de vida deberían ser desde la primera infancia.

\section{CONCLUSIONES}

Se concluye que la población de adolescentes escolarizados de Arica-Chile presentan valores antropométricos alterados con altos índices de sobrepeso y obesidad asociado a un riesgo metabólico de un $24,7 \%$. Las mujeres presentan mayores índices de alteraciones respecto de los hombres. Los parámetros más alterados de sobrepeso y obesidad se presentan en mujeres de segundo año de enseñanza media con porcentajes de $49,1 \%$ versus $33,8 \%$ en los hombres. Los índices de masa corporal (IMC), perímetro de cintura (PC) e índice cintura talla (ICT), presentan altas correlaciones $(r>0,5)$. Se sugiere enfocar las intervenciones educativas según sello de vida saludable, incentivando la actividad física y mejorar los hábitos alimenticios en los adolescentes.

AGRADECIMIENTOS. Este trabajo fue subvencionado por el proyecto UTA Mayor $\mathrm{N}^{\circ}$ 4716-17. También se agradece al Proyecto UTA-Escuela. Convenio Marco Formación Inicial Docente, UTA N ${ }^{\circ} 1856$ - 2018, Universidad de Tarapacá, Chile.

ESPINOZA-NAVARRO. O. \& BRITO-HERNÁNDEZ, L. Morphological patterns associated with metabolic risk factors in the adolescent school population. Int. J. Morphol., 38(6):1645-1650, 2020.

SUMMARY: The sustained increase in the prevalence of overweight and obesity in children and adolescents causes alarm in the scientific community, who observe significant increases in diseases associated with Metabolic Syndrome (MS), in adult life. Chile presents $75 \%$ with overweight and obesity in the adult population and $50.9 \%$ 
in the student population and with $66.2 \%$ of sedentary lifestyle. The objectives of this work were to determine the anthropometric profiles and their association with metabolic risk in adolescents from a subsidized private school in Arica-Chile. The total of high school students, $n=810$ (women $n=437$ and men $n=373$ ), were evaluated according to: weight, height, waist circumference, waist-height index (WHI), body mass index (BMI). Metabolic risk was calculated for all of them according to Ashwell \& Gibson protocols and BMI according to Quetelet's formula. The data were analyzed in statistics for central tendency, dispersion, and percentages. Pearson test ( $r>0.5)$ was used for correlation. The results show that $44.1 \%$ of women and $37.2 \%$ of men are overweight and obese. The general metabolic risk of the study population was $24.6 \%(24.7 \%$ and $24.4 \%$ in women and men, respectively). A high correlation was observed between WC / WHI ( $\mathrm{r}=$ $0.92)$, BMI / WHI $(r=0.86)$ and Weight / WC $(r=0.87)$. It is concluded that adolescents present altered anthropometric values that indicate high rates of metabolic risks. The most altered parameters were observed in the second year of secondary education with percentages of overweight and obesity of $49.1 \%$ in women versus $33.8 \%$ in men. Regardless, males presented better morphometric indices than females. High correlations $(r>0.5)$ were observed in all the parameters under study, associated with metabolic risk. It is suggested to focus educational interventions according to the seal of healthy living, encouraging physical activity and improving eating habits in school adolescents.

KEY WORDS: Education; Metabolic Risk; Adolescents; Sedentary Lifestyle; Chile.

\section{REFERENCIAS BIBLIOGRÁFICAS}

Acosta-García, E. \& Concepción-Páez, M. Índice cardiometabólico como predictor de factores de riesgo cardiovascular en adolescentes. Rev. Salud Pública, 20(3):340-5, 2018.

Ashwell, M. \& Gibson, S. Waist-to-height ratio as an indicator of 'early health risk': simpler and more predictive than using a 'matrix' based on BMI and waist circumference. BMJ Open, 6(3):e010159, 2016.

Atalah, S. E. Epidemiología de la obesidad en Chile. Rev. Med. Clin. Las Condes, 23(2):117-23, 2012.

Brito-Hernández, L.; Espinoza-Navarro, O.; Díaz-Gamboa, J. \& Lizana, P. A. Postural evaluation and prevalence of hyperkyphosis and hyperlordosis in students of elementary school. Int. J. Morphol., 36(1):290-6, 2018.

Burrows, R.; Correa-Burrows, P.; Reyes, M.; Blanco, E.; Albala, C. \& Gahagan, $\mathrm{S}$. High cardiometabolic risk in healthy Chilean adolescents: associations with anthropometric, biological and lifestyle factors. Public Health Nutr., 19(3):486-93, 2016.

Collipal, L. E. \& Godoy, B. M. P. Social representations of obesity in preuniversity and university youth. Int. J. Morphol., 33(3):877-82, 2015.

Cordero, M. R. E.; Rodríguez, A.; Hernández, C. I.; Méndez, E.: Pifano, C. \& Infante, B. Biomarcadores cardiometabólicos e indicadores antropométricos de adiposidad en adolescentes escolares. Vitae Acad. Biomed. Digit., (62), 2015. Disponible en: http://vitae.ucv.ve/ index_pdf.php?module=articulo_pdf $\& n=5122 \& r v=118$.

Díaz, D. O.; Hernández, R. J.; Domínguez, A. E.; Martínez, M. I.; Bosch, P. Y.; del Busto, M. A.; García, E. D. M. \& Rodríguez, F. L. Valor de corte de la circunferencia de la cintura como predictor de disglucemia. Rev. Cuba. Endocrinol., 28(1):1-15, 2017.

Espinoza-Navarro, O.; Brito Hernández, L. \& Lagos-Olivos, C. Body composition and metabolic risk factors in primary in primary school teachers in Chile. Int J. Morphol., 38(1):120-5, 2020.

Guszek, S.; Ciesla, E.; Guszek-Osuch, M.; Kosie, D.; Kiebzak, W.; Wypcho, $\&$ Suliga, E. Anthropometric indices and cut-off points in the diagnosis of metabolic disorders. PLoS One, 15(6):e0235121, 2020.

Huamán, J.; Alvarez, M.; Gamboa, L. \& Marino, F. Índice cintura-estatura como prueba diagnóstica del Síndrome metabólico en adultos de Trujillo. Rev. Med. Hered., 28(1):13-20, 2017.

Iglesias Diz, J. L. Desarrollo del adolescente: aspectos físicos, psicológicos y sociales. Pediatr. Integral, 17(2):88-93, 2013.

Leiva, A. M.; Petermann-Rocha, F.; Martínez-Sanguinetti, M. A.; TroncosoPantoja, C.; Concha, Y.; Garrido-Méndez, A.; Díaz-Martínez, X.; LanuzaRilling, F.; Ulloa, N.; Martorell, M.; et al. Asociación de un índice de estilo de vida saludable con factores de riesgo cardiovascular en población chilena. Rev. Med. Chile, 146(12):1405-14, 2018.

Macedo-Uchôa, F.; Pinheiro-Lustosa, R.; Cintra-Andrade, J.; NogueiraGodinho, W.; Marques-Aranha, A.; Deana, N. F. \& Alves, N. The influence of physical activity and eating behaviour on body mass index in children and adolescents: A review the literature. Rev. Chil. Nutr., 46(3):343-51, 2019.

Martínez-Villanueva, J. Obesidad en la adolescencia. Adolescere, (3):43-55, 2017.

Mera-Gallego, R.; García-Rodríguez, P.; Fernández-Cordeiro, M.; RodríguezReneda, A.; Vérez-Coltelo, N.; Andrés-Rodríguez, N. F.; Fornos-Pérez, J. A. \& Rica-Echevarría, I. Factores de riesgo cardiovascular en adolescentes escolarizados (RIVACANGAS). Endocrinol. Nutr., 63(10):511-8, 2016.

Ministerio de Educación (MINEDUC). Estadísticas de la Educación. Santiago de Chile, Centro de Estudios, Unidad de Estadísticas, Ministerio de Educación, Gobierno de Chile, 2018.

Ministerio de Salud (MINSAL). Encuesta Nacional de Salud, 2016-2017. Primeros resultados. Santiago de Chile, Departamento de Epidemiología, División de Planificación Sanitaria, Subsecretaría de Salud Pública, 2017.

Organización Mundial de la Salud (OMS). Panorama de la Seguridad Alimentaria y Nutricional en América Latina, 2016. Ginebra, Organización de las Naciones Unidas para la Alimentación y la Agricultura (FAO) y Organización Panamericana de la Salud (OPS), 2016.

Organización Mundial de la Salud (OMS). Salud de la Madre, el Recién Nacido, del Niño y del Adolescente. Ginebra, Organización Mundial de la Salud, 2014. Disponible en: https://www.who.int/maternal_child_adolescent/ topics/adolescense/dev/es/

Ramos-Padilla, P.; Carpio-Arias, T.; Delgado-López, V. \& Villavicencio-Barriga, V. Sobrepeso y obesidad en escolares y adolescentes del área urbana de la ciudad de Riobamba, Ecuador. Rev. Esp. Nutr. Hum. Diet., 19(1):21-7, 2015

Reynoso Vázquez, J.; Carrillo Ramírez, J.; Algarín Rojas, L.; Camacho Romero, O. \& Ruvalcaba Ledezma, J. C. La obesidad y su asociación con otras de las enfermedades crónicas no transmisibles. J. O. N. N. P. R., 3(8):558763,2018

Villegas-Sepúlveda, L.; Romo-Gasson, I.; Aveytia-Rojas, J. R. \& HernándezLoya, A. J. Determinación de sobrepeso y obesidad en adolescentes que ingresan a una secundaria privada en la ciudad de Chihuahua. Rev. Esp. Méd. Quir., 21(1):3-9, 2016.

\author{
Dirección para correspondencia: \\ Prof. Dr. Omar Espinoza Navarro \\ Facultad de Ciencias \\ Departamento de Biología \\ Universidad de Tarapacá \\ Avenida. General Velásquez Nº 1775 \\ Arica \\ CHILE
}

Email: omarespinoza091@gmail.com

Recibido : 24-07-2020

Aceptado: 14-08-2020 